\title{
An Improved Multi-Biometric System for Authentication
}

\author{
Om Prakash Sharma, Sivaramkumar P
}

\begin{abstract}
Biometrics is the new technology for calculating and measuring the body parts of a person. It is playing an important role in identifying an individual. It signifies a metrics related to person characteristics (physiological or behavioral). Biometric system may be based on single modal or multiple modals. Multimodal system also termed as multi-biometric system (hybrids two or more modals) are becoming popular. The idea behind the paper is to implement and improve the authentication processusing multiple trait for identifying a person. Here, the combination of Iris \& Fingerprint based biometric model is presented. The relevant features (key-points) are extracted from these two traits in parallel and then they are passed through matching module. The key-points are extracted using Discrete Wavelet Transform (DWT) and Speeded-Up Robust Feature (SURF) descriptor and then passed to the matching (mapping) module where mapping is done using the Normalized Weighted Sum-Rule. The experimental result showed that the proposed multi-biometric model performs well showing clear variation in FAR and FRR against the existing models.
\end{abstract}

Keywords: Biometric, multimodal, fingerprint, iris, authentication, recognition.

\section{INTRODUCTION}

The current world trend has moved daily life security. It is a key issue of the life science. Today in this modern era biometric based security system is one among those systems which is more secured than other security system based on Password, Pin, and ATM card etc. In general biometric is used for an authentication purpose. The term biometric has root in the Greek word 'bios' meaning 'life' and 'metrikos' means 'to measure'. It refers to technologies that scrutinize and measure human physiological cum behavioral characteristics for the authentication purposes. The physiological features include (fingerprint, iris, face etc.) and behavioral features include (voice patterns, signature, writing styles, gait etc.) [1]. Any multi-biometric system is basically designed to function in two modes- the first one is an enrolment mode and the second is the detection/recognition mode [2]. During enrolment the image is captured using sensor(s) as an input and then it is passed through intermediate stages of image processing to obtain image templates and these are finally stored in the database for further references. During detection the input image is matched with the stored templates to obtain the scores on the basis of which the decision is made about an object is valid or invalid.

Revised Manuscript Received on December 30, 2019.

* Correspondence Author

Om Prakash Sharma, Assistant Professor to the School of IT, SRM University Sikkim.

Sivaramkumar P, Assistant Professor in School of Information Technology, SRM University, Sikkim

(C) The Authors. Published by Blue Eyes Intelligence Engineering and Sciences Publication (BEIESP). This is an open access article under the CC BY-NC-ND license (http://creativecommons.org/licenses/by-nc-nd/4.0/)
Today, usable biometric systems exist, including laptops having thumbprint, facial recognition, similarly palm print systems for secure admission into constrained region, the use of fingerprints to open car doors, and so on. However given the importance of biometrics, and the well-known drawback of using password-based authentication - it's very surprising that biometrics is not more widely used. For an ultimate biometric system, the following attributes and characteristics needs should be satisfied [1].

\section{A. Universal}

A biometric should be accepted virtually by everyone. In reality, no biometric trait is applicable to mass. For example, a small proportion of people do not have legible fingerprints.

\section{B. Distinguishing}

Each personality biometric should make a distinction with other entity. In reality, we can't expect a 100\% certainty, although, in theory, some methods can be distinguish with very low error rates.

\section{Permanent}

Ideally, the material characteristic being calculated should by no means change. In practice, it's adequate if the feature remains firm over a reasonably extended period of time.

\section{Collectable}

The physical attribute should be trouble-free to collect without causing any probable threat or harm to the subject of interest. To put into practice, collectability regularly depends greatly on how co-operative the subjects or entitiesare or not.

\section{E. Reliable, Robust, and User-Friendly}

These are just some of the additional attributes a biometric system should have to cope-up with real-world implementation. Some of the biometrics that has shown a promising result in practical environment or conditions has subsequently failed to carry comparable performance in practical. For any system to recognize accurately it must have capability to minimize intra-class variability and limit the inter-class variability. It's become easy for a system to reliably classify one object from the group of objects, if and only if when the variability among different instances of a given class is less than the variability between different classes [1]. Iris having the most discriminate texture and fingerprint being the globally accepted and popular, is used to form a model of the system. The exploitation of Biometrics for authentication and recognizing individuals from the mass has emerged as one the most convincing and reliable method. 


\section{An Improved Multi-Biometric System for Authentication}

For biometrics to be a realistic substitution for passwords or any other traditional system for authentications, economical and trustworthy systems are needed.

Most of the extensively deployed Fingerprint cum Iris based biometric systems today use information from an individual trait or individual biometric characteristic for itsverification or identification. Many single biometric techniques have to deal with other difficulty such as population and demographic diversity, varied utilization atmosphere, and a lot of exacting performance necessities. Existing Multi-Biometric verification technology measure finding it tough to fulfil these demands and an answer is to integrate extra sources of knowledge to strengthen the choice method.

In this paper bimodal scheme based on fingerprint \& iris (FIMBAS) is discussed. First the pre-processing and segmentation of iris image is done to obtain iris template. Then next the two dimensional discrete wavelet transform (DWT) is applied on Iris template and on the resultant of it at two level, SURF is applied on Approximate band (LL) of DWT to obtain SURF Descriptors which results an Iris features vector. In parallel the pre-processing of fingerprint is also performed to obtain region-of-interest (ROI) points. The powerful SURF algorithm (from Bay and Tuytelaars) is again applied on Approximate band (LL) of DWT to obtain SURF Descriptors which results in feature vector of Fingerprint. The Features obtained are invariants to scale, rotation and illuminations.

Once the features are computed for iris cum fingerprint both, they are matched independently against the enrolled templates in the database. The test image and database image features vector are compared using simple and popular distance measuring scheme, Euclidean Distance (ED) for matching. The result obtain is pass through normalization process using Min-Max normalization techniques to bring the values in between 0 and 1 . Then mean function is applied to obtain generalized single value, finally the value computed for both the traits gets fused using weighted-sum-rule and the result obtain is compared against the preset threshold value.

\section{BACKGROUND}

On the basis of various research works and finding large number of model have been proposed in fields of biometric system. These systems have been developed mainly for uni-modal system comprising of fingerprint or many other traits in individual like iris including many other traits. The word biometric derived from Greek word is used for wide range of application domain with various purposes and meanings. A brief background on how to use of iris cum fingerprint is presented below.

The idea of opting for iris for recognizing persons was suggested long before in the year 1936 by U.S. ophthalmologist named Frank Burch but then no sign of technology was there for making it feasible. Finally, after a long research gap US born mathematician name John Daugman's ( a professor at Cambridge University, England) and his co-partner Flom and Safir in 1994 develop a path breaking algorithms ( based on mathematical processes) that can turn images of irises into unique feature codes. Daugman's was given patent \#5,291,560 in U.S. for his work-"iris based personal identification system" the same year. His work was widely accepted as the creator of true recognition system using iris. Most of iris-scanning systems today use his algorithm.

Next, the oldest and most popular in use among all trait; fingerprint has very ancient history. During 2,000 B.C. the Babylonians depends on the use of fingerprints to sign on contracts and business proposals to help overcome the forgery. Apart from that the sign of usage and facts of fingerprint was observed in various countries for various different applications. After a long gap in 1880 Henry Faulds, (surgeon) functioning in Tokyo published a piece of writing describing a system which could recordfingerprints using ink for criminal identification. But his idea was summarily rejected by London's Metropolitan Police. Then again after a span of time in 1897 in Calcutta, (what is known as Kolkata today), Officers Hem Chandra Bose and Azizuln Hague develop the "Henry System" can be used for fingerprint classification (under the supervision of, Sir Edward Richard Henry) and the first case using their system against a criminal conviction was reported in Bengal, in 1898. Finally after a long research gap in the year 1981 first five AFIS deployed as a part of project funded by FBI to make fingerprint recognition an automated process. Then the year 1999 onwards the commercial products based on fingerprint became popular as medium of authentication in the market for controlling access of vital resources, log on and security checks.

\section{RELATED WORKS}

This David Zhang et. al. suggested an approach based on neural network for classification face and palm-print together as a recognition model. The matching scores obtained in individual from face and palm-print for same person are used as inputs to train a system using feed-forward neural (FFN) network [5].

In 2005, Jain et al. proposed a multimodal technique that uses face, fingerprint and hand geometry feature scores for fusion. The matching of modalities is done using matcher Euclidean distance [3] [4].

In 2006, Li et al. proposed a new feature finding for hand geometry, which is a amalgamation of hand shape, palm-print and knuckle print. The features were integrated from three biometrics using fusion at feature level. Feature set are obtain using Kernel Principle Component Analysis (an amalgamation of kernel projection and PCA for dimension reduction) [6].

Again in 2007, Li et.al introduced a model based on Linear Discriminant Analysis covering Full space (FSLDA) using ear, face and combined ear cum face images for recognition [7].

In 2008, Nandakumar et al. introduced a multimodal system based on fusion done at matching score level. They designed a framework which could optimally merge the matching scores on the basis of likelihood ratio test [8].

In 2009, P. Aruna et al. [9] proposed a theoretical approach for person authentication using multiple biometrics. The feature from fingerprint, palm-print and iris is fuse at feature level. Biometrics trait like iris being an internal part of human is less prone to spoil. The proposed system uses Gabor texture as feature points from pre-processed iris, palm-print and fingerprint image. 
These features are then fused using a

wavelet based feature combining technique. Since the features obtained from these biometric traits are in different sizes, hence the system uses discrete wavelet-transform based fusion techniques at fusion level in amalgamation with PCA \& ICA.

In 2014, Benaliouche and M. Touahria; experimented the comparative analysis of three different approaches based on

multimodal scheme for recognition by combining iris \&fingerprints using classical sum-rule, weighted sum-rule, and fuzzy logic [10]. The matching scores from different biometric traits were fused at the score level and at the decision levels. The scores amalgamation approach was done after normalization of scores from both the modalities using the min-max rule. Experimental results concluded that the fuzzy logic applied on the matching scores at the decision level gave the best result in comparison to the classical sum-rule \& weighted sum-rule in order

In 2015, Divyakant T. Meva, et. al. [11] presents multimodal system that improves the success rate of verification. They have adopted fingerprint and face for modality to match the inputs applying the fusion at score level. They have tried to identify accomplishment rate using various permutation of weights assigned to match scores from individual traits.

\section{MULTI-BIOMETRIC SYSTEM}

Model using an amalgamation of two or more biometric modal or algorithm for the purpose of authentication as in Figure 1 is called multi-biometric system [4].

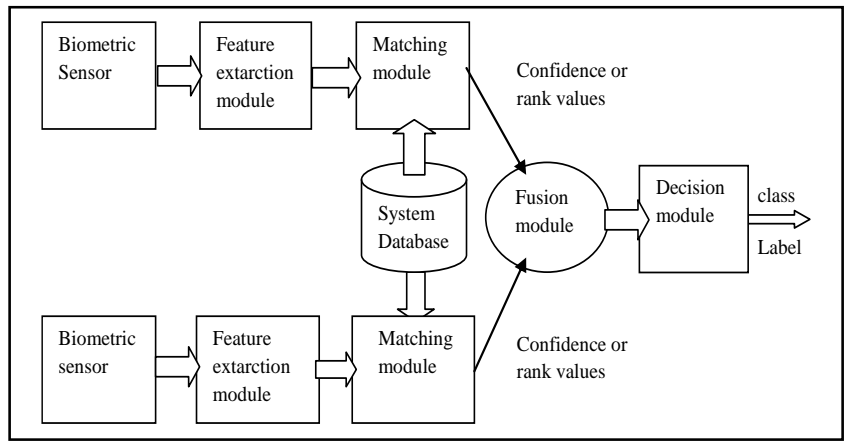

Figure 1: Rank based Multimodal Biometric System

The lower accuracy rate and trustworthiness of established authentication system and unimodal systems has pushed numerous end users to opt for multimodal systems as it provides a highest level of accurateness during authentication process. Most of the unimodal systems often face major challenges due to susceptible to noise, intra-class unevenness, data value, non universality, spoofing attack and many other factors. These restrictions on unimodal systems can be overcome by using multimodal systems. Overall the multi-biometric system reduces False Rejection Rate (FRR) \& False Acceptance Rate (FAR). A multi-biometric system overcomes the stated restrictions of unimodal by providing information on multiple evidences of the identical personality. These models help to achieve an enhanced performance that may not be feasible relying just on a particular biometric trait..

\section{METRICS AND MEASURES}

The performance any biometric model depends upon the computational assessment on accuracy and other quantifiable characteristics of the system. None of the metrics are adequate enough to provide trustworthy and desired [2]. The decision from biometric system about an individual's is either an impostor or genuine type decision can be represented by two numerical distributions called impostor distribution \& genuine distribution, respectively. For each type of result, there are two possible solutions, true or false, in total there are four potential outcomes [2]:

- a genuine character is accepted.

- a genuine character is rejected.

- an impostor is rejected.

- an impostor is accepted.

In general, False Acceptance Rate (FAR) is defined as the rate of acceptance of an impostor and is computed as:

$$
\text { far }=\frac{\text { nos of imposter accepted } * 100}{\text { total nos of imposter compared }} \%
$$

Next the False Rejection Rate (FRR) defined as the rate of rejection of genuine person is computed as:

$$
\text { far }=\frac{\text { nos of genuine person rejected } * 100}{\text { total nos of genuine compared }} \% \text { (1.2) }
$$

And finally the Equal Error Rate (EER), the value at which the FAR and FRR are equal is computed as:

$$
\text { err }=\text { far for which far }=\text { frr }
$$

\section{RESEARCH METHODOLOGY}

Fig.2 shows the block diagram of FIMBAS system. It includes different stages of proposed multi-biometric recognition system and the brief descriptions on important steps are given as- Feature selection itself is one of the most important factors to contribute high performance and high accuracy in recognizing the objects or traits by the system. Researchers have contributed various techniques and tools for feature extraction. Among these tools and techniques, the recent and perhaps the interesting algorithms are Discrete Wavelet Transform (DWT), Scale Invariant Feature Transformation (SIFT), Speedup Robust Feature (SURF), and various versions of FAST [12]. The Experimental result presented in [13] confirms that the DWT and SURF technique employed for feature extraction module outperforms the various limitation of SIFT and FAST. The resultant analysis is an outcome from an implementation of various stated feature extraction tools on two differentbiometric traits (eye, fingerprint). The implementation also showed that SIFT being powerful tools fails in some cases in extracting the relevant features compare to SURF. 


\section{An Improved Multi-Biometric System for Authentication}

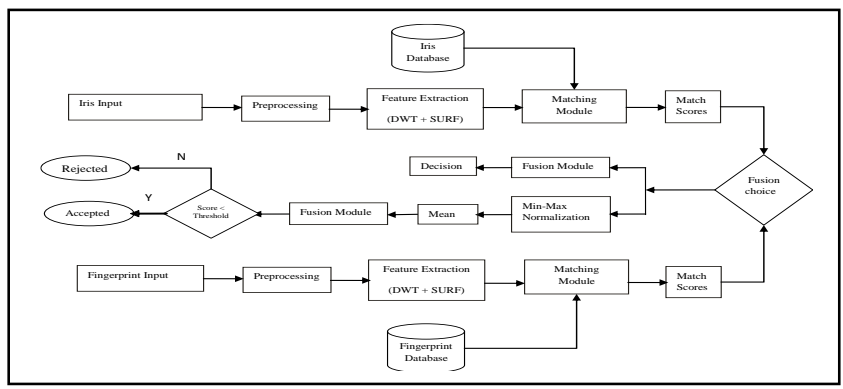

Fig.2 Block diagram of FIMBAS system (Horizontal View)

\section{A. Speeded Up Robust Features (SURF)}

Surf is a novel approach to interest point detector and descriptor that are invariant to scale and rotation introduced by Bay and Tuytelaars in 2006. It approximates or even outperforms the existing algorithms such as SIFT and FAST with respect to invariability, distinctiveness, and robustness. Here the key-points are computed and extracted at much faster rate in comparison to SIFT because of the use of integral images for convolutions, a Hessian matrix for detector and distribution of Wavelet responses as descriptor [14][15]. The two main steps in SURF are:

- Interest Point Detection: The detection is carried out using Hessian matrix based measure to detect interest points in an image which are invariant to scale and rotation.

- Interest Point Descriptor: A distribution of Haar-wavelet responses within a local region are used as descriptor.

\section{B. Discrete Wavelet Transform (DWT)}

The technology is based on sub-band coding; it can perform a fast computation of Wavelet Transform. Discrete Wavelet Transform is easy to implement and has been seen that it reduces the computation time at great level for finding approximation and other details. According to S. Mallet [16], the Discrete Wavelet Transform (DWT) can be implementedusing filter banks involving Low-Pass (LP) and High-Pass (HP) filters as shown in the fig. 4. The DWT is carried out on rows first (i.e. row by row) and then column by column. As a result, four sub images are generated.

- The top left section corresponds to sub image which gives an approximation of the originalimage.

- The top right section corresponds to sub image which gives the horizontal details.

- The bottom left section corresponds to sub image which gives the vertical details.

- The bottom right section corresponds to sub imagewhich gives the diagonal details.

The decomposition procedure is further iterated on the resulting approximation sub image as shown in Fig. 7 and Fig. 8 shows a two-level decomposition of the colourimage for iris and fingerprint.Based on the above affirmation, the multiple transformations function such as DWT and SURF are used in the proposed system (FIMBAS) to generate features vector from fingerprint cum iris. These features are matched independently against their templates stored in the database during enrolment phase. The test image features and features templates in the database are compared by using Euclidean Distance (ED) for matching. The results obtained in terms of scores is pass through mean function to obtain generalized single value, after that normalization is done on the mean score using Min-Max normalization techniques to bring the value in a range between 0 and 1 . Finally the result below threshold value is considered as optimally and accurate matched with target. It is noticed that the GAR, FRR and FAR values are better in the proposed technique compared to existing techniques.

\section{WORKING OF THE MODEL}

Most of the image processing system goes through the same processes/phases in order to recognize the images given as input by matching it against the existing image templates enrolled in the database. Similarly biometric being based on same technology employ the same basic processes except the different approaches (algorithms) at different stages of image detection. The important and most abstract representations of phases are depicted in the proposed scheme shown above in fig.2.

\section{A. Image Acquisition}

The required biometric traits are captured in this phase using appropriate capturing devices and sensors. The different image sensors are digital cameras, mobile cameras and web cameras etc. The number of samples is considered and database is created. The database comprises of more number of biometric samples collected from different image sensors in different sessions and time, with different intensity of light, at different angles, expressions and impressions. Among the popular database for fingerprints, FVC2004 and FVC2006 are standard on which test can be implemented robustly. For iris, the biometric databases are viz., UBIRIS and CASIA etc.

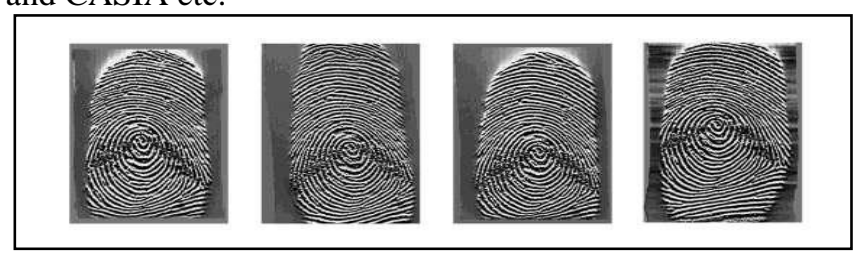

Fig.3: Samples of Fingerprint

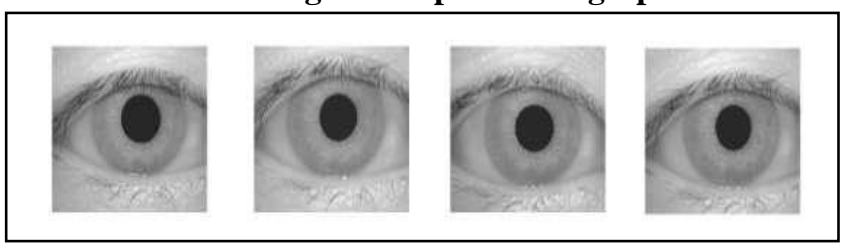

Fig.4: Samples of Eye image

\section{B. Pre-processing \& Segmentation}

After pre-processing is done segmentation process starts. In segmentation actual iris part is isolated from a noise free eye image. Segmentation plays an important role in image detection system. Properly segmented images helps in addition of relevant and key features points from an image which further helps in detection process accurately minimizing the FAR and FRR. At the end of segmentation process eyelids cum eyelashes occluded in the upper parts and lower parts of iris region are removed along with specular reflections within the iris section that may distort the iris pattern. The result of pre-processing and segmentation is presented in a Fig.5. \& Sciences Publication 


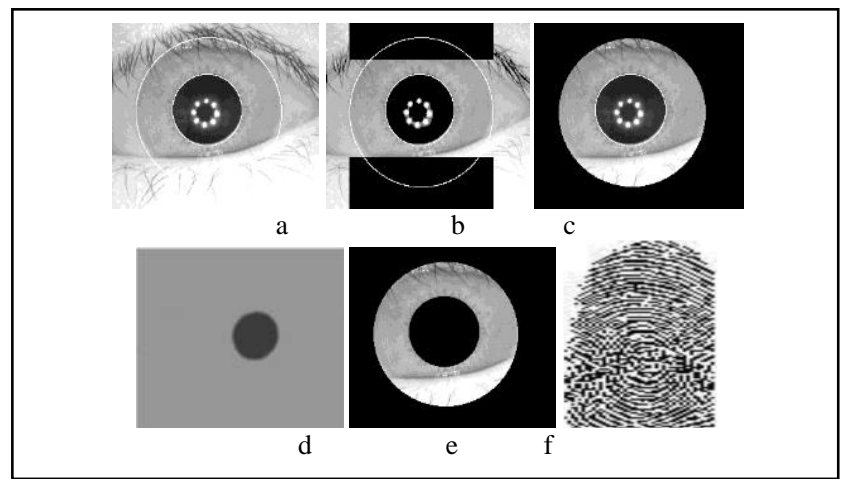

Fig.5: The result of pre-processing \& segmentation process

After pre-processing is done segmentation process starts. In segmentation actual iris part is isolated from a noise free eye image. Segmentation plays an important role in image detection system. Properly segmented images helps in addition of relevant and key features points from an image which further helps in detection process accurately minimizing the FAR and FRR. At the end of segmentation process eyelids cum eyelashes occluded in the upper parts and lower parts of iris region are removed along with specular reflections within the iris section that may distort the iris pattern. The result of pre-processing and segmentation is presented in above Fig. 6. Here each image represents:

Iris \& Pupil Detection- using Canny Edge detection and Hough transform techniques.

a) Masking the Noise value as NAN- using Libor Masek Code.

b) Iris \& Pupil Segmentation - using Masking technique.

c) Pupil Segmentation - using Masking technique

d) Segmented iris - using difference of c \& d techniques.

e) Segmented fingerprint - using thinning and cropping.

\section{Feature Detection}

The DWT is put into function on iris \& fingerprint template of given size to produce four sub-bands such as $\mathrm{HH}, \mathrm{HL}, \mathrm{LH}$ and LL given in Fig. 6\&7.

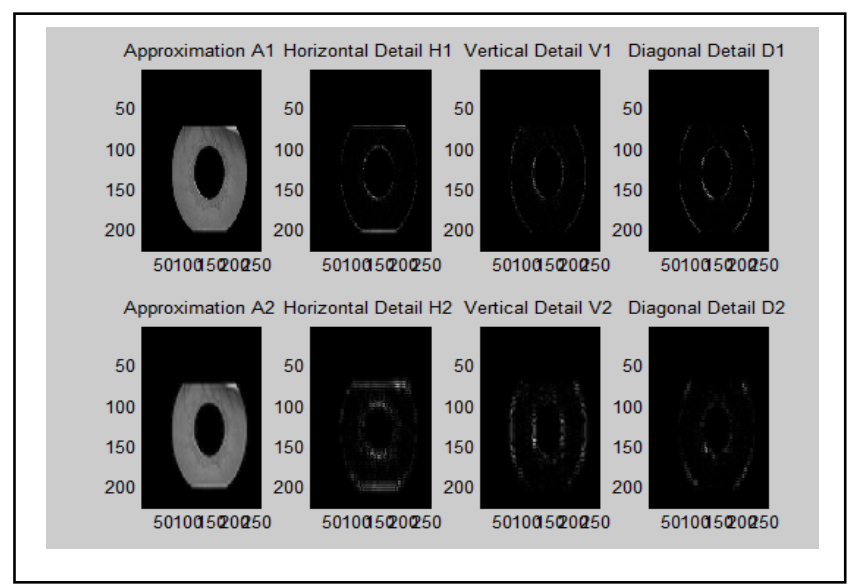

Fig. 6: Iris approximation bands at two levels

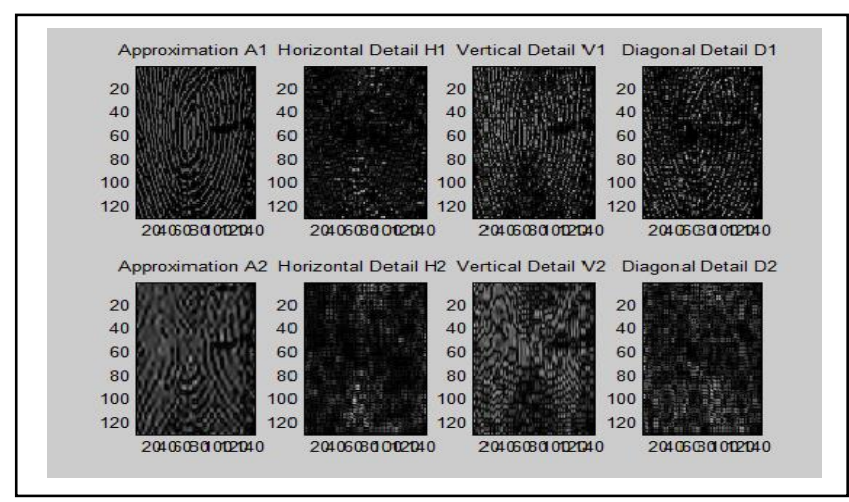

Fig.7: Fingerprint approximation bands at two levels

SURF is put into function on LL band (approximation band) of decomposed image to generate SURF descriptors. The DWT sub-bands and SURF descriptors of an images is given in Figure 8. The absolute resultant value of SURF descriptors obtain form an iris features vector.

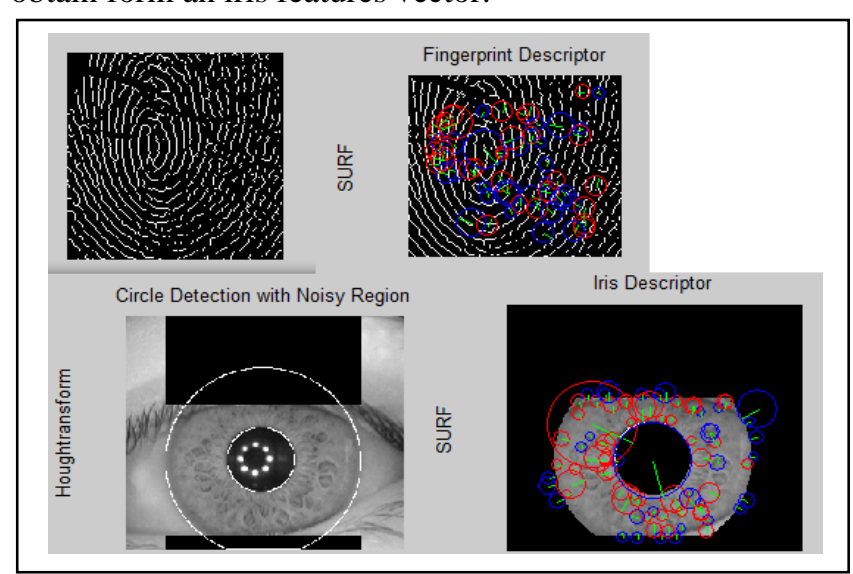

Fig.8: Fingerprint \& Iris Feature Descriptors'

\section{Fusion Methodology}

In this system the matching distance calculated for each modality is passed through the normalized module. The normalized rule brings the matched score in between 0 and 1 .

$$
\begin{aligned}
& N_{\text {iris }}=\frac{M S_{\text {iris }}-\min _{\text {ivis }}}{\max _{\text {ivis }}-\min _{\text {ivis }}} \\
& N_{\text {finger }}=\frac{M S_{\text {fingey }}-\text { min }_{\text {finger }}}{\max _{\text {finger }}-\text { min }_{\text {finger }}}
\end{aligned}
$$

where $M S_{\text {Iris }} \& M S_{\text {Finger }}$ are matching scores realised from iris \& fingerprint modalities, $\min _{\text {Iris } \& \max _{\text {Iris }} \text { are the minimum }}$ score and maximum score for iris detection and $\min _{\text {Finger }} \&$ max $_{\text {Finger }}$ are corresponding result (values) from fingerprint. Apart from these other normalization procedures also exist, like $Z$-score, Sigmoid \&TanH procedure which gives optimal results. TanH method gives the optimum result as it includes a numerous parameters. Z-score \& Min-Max are easy and simple to implement but they are more prone to presence of outliers [17]. The result obtained for each modality is then fused using weighted sum-rule and on account of outcome after fusion, the decisionis taken comparing to the ser threshold value. Depending upon an 


\section{An Improved Multi-Biometric System for Authentication}

accuracy level required by the system, the threshold value is set dynamically. These threshold values are decided after an extensive experimental result.

$$
S^{\prime}=\alpha N_{\text {iris }}+(1-\alpha) N_{\text {finger }}(1.5)
$$

\section{E. Matching}

The probability of matching a person with a different person is high with single biometric such as iris or fingerprint. The fusion of two biometric trait parameters reduces the probability of matching with different persons. The features of iris \& fingerprint are fused using dynamic weighted sum-rule to generate final set of feature vector for accurate detection of person. The ED is used for the comparison between final feature vectors set of database image with feature vector set of test images for matching.

$$
E D=\sqrt{\sum_{i=1}^{n}\left(X_{i}-Y_{i}\right)^{2}}
$$

\section{EXPERIMENTAL RESULTS}

The matching procedure are implemented using normalized weighted sum-rule where the weight value is assigned dynamically based on condition laid for fusion technique for both modalities (iris \& fingerprints). Figure 9 presents the graphical user interface showing the detection done using the normalized weighted sum-rule. The normalization of score is done before fusion using the min-max rule. In this work forexperimentation the weight value is set as w1 to 0.5 for the iris and $w 2=(1-w 1)=0.5$ for the fingerprint (when both the images are found matched), but in case if any one missed the target threshold then the weight are dynamically assigned, for matched it is 0.2 and for unmatched trait 0.8 .

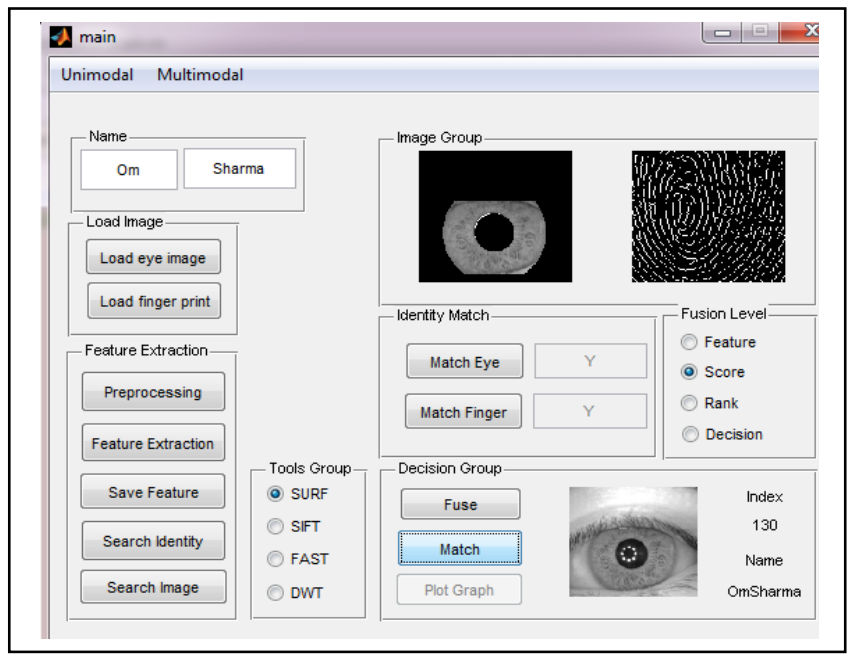

Fig. 9: GUI shows match result at Score Level

\section{PERFORMANCE ANALYSIS}

This section presents the experimental performance analysis of iris \& fingerprint based multi-biometric system. MATLAB with Windows operating system is utilized to perform the experiments. The computer used was equipped with an Intel Core i3-4430s (HpProBook) with $2 \mathrm{GHz}$ (Gigahertz) CPU (Central Processing Unit) and 4 GB of RAM (Gigabytes of Random Access Memory).
The proposed Multi-biometric model was investigated about the efficiency, robustness against various authentication systems. The dataset from CASIA V2.0 for iris dataset and FVC2004 DB3_A for fingerprint dataset were used. The multi-biometric scheme using fusion gives better results in comparison using a single trait either iris or fingerprint in individual. Matching using the normalized weighted sum-rule where weight is dynamically assigned and adjusted provides superior identification results compared to the conventional weighted sum-rule with static weight assigned in order.

Table 1: shows the percentage of FRR values decreases from $100 \%$ to $0 \%$ as threshold increases. It is optimum at 0.55 i.e. 2\% FRR. The GAR and FAR values are increased with increase in threshold values, at 0.055 it is $98 \%$ GAR and $0 \%$ FAR in the case of fusion of score from different modality. ERR is $2 \%$.

Table 1: Percentage variations of FRR, GAR and FAR values against Threshold for Fusion

\begin{tabular}{|c|c|c|c|}
\hline Threshold & \% FRR & \%GAR & \% FAR \\
\hline 0.005 & 70 & 10 & 0 \\
\hline 0.015 & 55 & 65 & 0 \\
\hline 0.025 & 35 & 75 & 0 \\
\hline 0.035 & 15 & 85 & 0 \\
\hline 0.045 & 5 & 95 & 0 \\
\hline $\mathbf{0 . 0 5 5}$ & $\mathbf{2}$ & $\mathbf{9 8}$ & $\mathbf{0}$ \\
\hline 0.065 & 0 & 98 & 15 \\
\hline 0.075 & 0 & 100 & 35 \\
\hline 0.085 & 0 & 100 & 65 \\
\hline
\end{tabular}

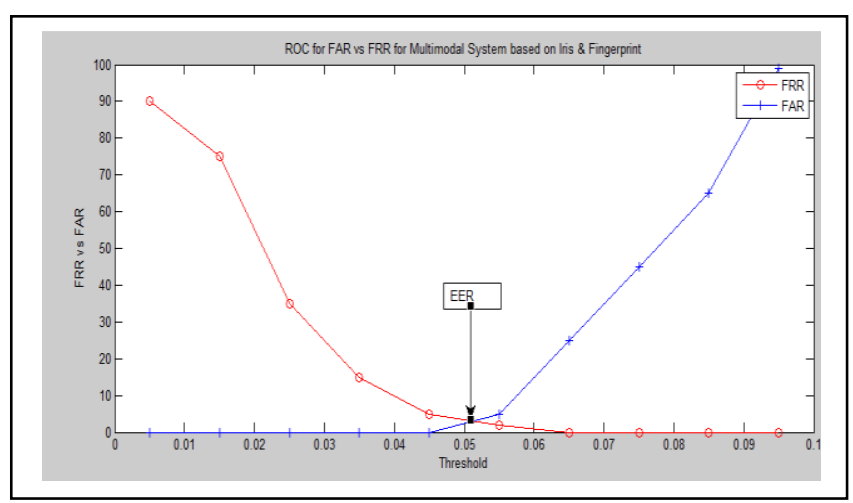

Fig. 10: ROC for FRR \& FAR vs. Threshold

The Table 2: shows the percentage FAR \& FRR values for existing scheme and proposed scheme. It is noticed that the valves of FAR \& FRR are less in the proposed scheme compared to existing scheme (model) presented by Abdolahiet. al.[7]. Kankrale and Sapkal [20], and many other researcher like AsimBaig et al.[18], FilippoSorbello Vincenzo Conti [19]. Hence the proposed modal using DWT and SURF descriptor is better compared to existing algorithms as illustrated in table below.

Table 2: shows the percentage of FAR \& FRR values for existing scheme and proposed scheme.

\begin{tabular}{|l|l|l|l|c|}
\hline Model & $\begin{array}{l}\text { Feature } \\
\text { Extraction } \\
\text { Method }\end{array}$ & $\begin{array}{l}\text { Level for } \\
\text { Fusion }\end{array}$ & $\begin{array}{l}\text { \% } \\
\text { FAR }\end{array}$ & $\begin{array}{c}\text { \%RR } \\
\text { FRR }\end{array}$ \\
\hline
\end{tabular}




\begin{tabular}{|l|l|l|c|c|}
\hline $\begin{array}{l}\text { AsimBaig et } \\
\text { al., [15] }\end{array}$ & Not Specified & Score level & 8.00 & 8.00 \\
\hline $\begin{array}{l}\text { FilippoSorbell } \\
\text { o Vincenzo } \\
\text { Conti [16] }\end{array}$ & Not Specified & $\begin{array}{l}\text { Feature } \\
\text { level }\end{array}$ & 0.00 & 7.28 \\
\hline $\begin{array}{l}\text { Kankrale and } \\
\text { Sapkal [17] }\end{array}$ & $\begin{array}{l}\text { Minutia based } \\
\text { extractor + } \\
\text { Daugman's iris } \\
\text { extractor }\end{array}$ & $\begin{array}{l}\text { Feature } \\
\text { level }\end{array}$ & 0.00 & 5.12 \\
\hline $\begin{array}{l}\text { Gawande et } \\
\text { al. } \\
\text { 2012 [20] }\end{array}$ & $\begin{array}{l}\text { 1D log Gabor filter } \\
\text { for both modalities }\end{array}$ & $\begin{array}{l}\text { Feature } \\
\text { level }\end{array}$ & 0.00 & 2.00 \\
\hline $\begin{array}{l}\text { Abdolahi et } \\
\text { al. 2013 [7] }\end{array}$ & $\begin{array}{l}\text { Modified minutia } \\
\text { based extractor + } \\
\text { Masek iris } \\
\text { extractor }\end{array}$ & $\begin{array}{l}\text { Decision } \\
\text { Level }\end{array}$ & 2.00 & 2.00 \\
\hline $\begin{array}{l}\text { Benaliouche } \\
\text { and Touahria, } \\
\text { 2014, [7] }\end{array}$ & $\begin{array}{l}\text { Minutia based } \\
\text { extractor + } \\
\text { Daugman's iris } \\
\text { extractor }\end{array}$ & $\begin{array}{l}\text { Decision } \\
\text { Level }\end{array}$ & 0.00 & 2.00 \\
\hline $\begin{array}{l}\text { Proposed } \\
\text { Model } \\
\text { (FIMBAS) }\end{array}$ & $\begin{array}{l}\text { DWT and SURF } \\
\text { descriptor + Masek } \\
\text { Iris extractor }\end{array}$ & Score Level & 0.00 & 2.00 \\
\hline
\end{tabular}

\section{CONCLUSION}

The objective of doing this research work is to design a process for drawing out key features from a mixed model and then improve it furtherusing best matching module. The biometric trait used in the work is iris cum fingerprint to achieve best negotiation between a zero FRR and its corresponding FAR. The table 2 shows performance comparison of various single biometric and multi-biometric system designed and developed by the researcher.

To improve the achievement rate of multi-biometric system, the various researcher designed biometric system with dynamic weight adjustment. The researcher has achieved significant improvement in successful identification / verification rates. In the proposed system too, the dynamic weight value has been assigned with little difference. If both the input trait gets matched individually the weight value is assigned in ratio 50:50, but in case of alternate situation where one of the trait fails to match then the highest ratio value is assigned to it (in ratio 0.8:0.2 or vice versa).

The highest success rate in the case of multi-biometric based system described in above gives 99\% (in approx.) accuracy. From the table 2 we can see further the sign of an improvement about $2-4 \%$ when successfully the verification process is implemented with the new approach. The fusion in multi-biometric gives superior results than using a mono-modal (single) based matching module for iris or fingerprint. Matching using the normalized weighted sum-rule where weight is dynamically assigned and adjusted provides enhanced recognition results compared to the classical weighted sum-rule with static weight assigned in order.

The research domain of multi-biometric system is vast containing various modalities. The research can even be continued with other modalities. The developed system is open for improvement by using the concept of learning. Like the expert system, where self-learning capabilities are there, the designed system can be embedded with learning module. Here the system can record weights of last successfully verified person respectively. For each user, iris cum fingerprint weights can be stored and on successful verification, these weights can be changed if they are adjusted during verification process. The system will learn itself. This method can also minimize the time consumption as weight adjustment cycles can be reduced significantly.

\section{ACKNOWLEDGMENT}

It gives immense pleasure to express the deep sense of gratitude to supervisor Dr. Praveen Srivastava \& Dr. Balamurugan B for their support and guidance. At the same time, thanks to Libor Masek\&Koveski for providing resource full code which could be exploited to complete the implementation. Finally thanks to Benaliouche and Touahria for writing paper which cleared various background concept on fusion strategies.

\section{REFERENCES}

1. Kresimir Delac and Mislav Grgic, "A Survey of Biometric Recognition Methods", International Symposium on Electronics in Marine, 2004, pp (184-193).

2. Surya Prakash and Phalguni Gupta, "Human Recognition using 3D Ear Images", Neurocomputing (2014), http://dx.doi.org/ 10.1016/j.neucom.2014.03.007, Elsevier.

3. Anil K. Jain, "Fundamentals of Digital Image Processing", Prentice Hall Information and System Sciences, Editor- Thomas Kailath.

4. [Anil K Jain, Arun Ross, and Salil Prabhakar, "An Introduction to Biometric Recognition," IEEE Transactions on Circuits and Systems for Video Technology, Vol.14, 2004, pp (1-29).

5. Zhang D. et. al, "Advanced pattern recognition technologies with applications to Biometrics". Medical Information science Reference, 2009-books.google.com.

6. SheebaJeya Sophia S. and Veluchamy S, "Security System Based on Iris Recognition", Research Journal of Engineering Sciences, ISSN 2278 - 9472, Vol. 2(3), 16-21, March (2013).

7. Li et.al."Multimodal Recognition Based On Face And Ear", IEEE Proceedings of the 2007 International Conference on Wavelet Analysis and Pattern Recognition, Beijing, China, 2-4 Nov. 2007.

8. Nandkumar et.al. "Multibiometric Systems: Fusion Strategies and Template Security", A Dissertation Submitted to Michigan State University in partial fulfillment of the requirements for the degree of Doctor of Philosophy, Department of Computer Science and Engineering 2008.

9. P. Aruna, K. KumariI and G. JayaSuma, "A Novel Multimodal Biometric Scheme For Personal Authentication", International Journal of Research in Engineering \& Technology, ISSN(E): 2321-8843; ISSN(P): 2347-4599, Vol. 2, Issue 2,pp. 55-66, Feb 2014.

10. Benaliouche et.al."Comparative Study of Multimodal Biometric Recognition by Fusion of Iris and Fingerprint", Hindawi Publishing Corporation of Scientific World Journal Volume 2014, Article ID 829369, 13 pages http://dx.doi.org/10.1155/2014/829369

11. Divyakant T. Meva, Dr C. K. Kumbharana, "Design and evaluation of multimodal biometric system with fingerprint and face recognition", International Journal of Scientific and Research Publications, Volume 5, Issue 4, April 2015, ISSN 2250-3153.

12. K. Delac and M. Grgic, "A survey of biometric recognition methods", In International Symposium Electronics in Marine, ELMAR, (2004).

13. D. Lowe, "Distinctive Image Features from Scale-Invariant Keypoints", International Journal of Computer Vision 60(2): 91-110, 2006.

14. H. Bay, T. Tuytelaars, and L. V. Gool, "SURF: Speeded up Robust Features", Journal of Computer vision and image understanding 110 (3): 346-359, 2008.

15. P Sharma and J Sheetlani, "Biometric based authentication system: a survey", International Journal of Current Advanced Research Volume 6; Issue 7; July 2017; Page No. 4487-4492

16. P Sharma, J Sheetlani and P Shrivastava, "Recent Advancement in Feature Extraction tools for Biometric System: Comparative Analysis",International Journal of Computer Sciences and Engineering Vol.7(2), Feb 2019, E-ISSN: 2347-2693, DOI: https://doi.org/10.26438/ijcse/v7i2.4650 | Available online at: www.ijcseonline.org

17. Hemanta Saikia and Kanak Chandra Sarma, "Approaches and Issues in Off-line Signature Verification System", International Journal of Computer Application, Vol. 4, pp (45-52), 2012. 


\section{An Improved Multi-Biometric System for Authentication}

18. Asim Baig, Ahmed Bouridane, Fatih Kurugollu, and Gang Qu, "Fingerprint Iris Fusion Based Identification System Using A Single Hamming Distance Matcher", International Journal of Bio-Science and Bio- Technology, Vol.1, 2009, pp (47-57).

19. Filippo Sorbello Vincenzo Conti, Carmelo Militello and Salvatore Vitabile, "A Frequency-Based Approach for Features Fusion in Fingerprint and Iris Multimodal Biometric Identification Systems", IEEE Transaction on Systems, Man, and Cybernetics", Vol. 40, 2010, pp (384-395).

20. R N Kankrale and S D Sapkal, "Template Level Fusion of Iris and Fingerprint in Multimodal Biometric Identification Systems," International Journal of Computer Applications, 2011, PP (18-24).

\section{AUTHORS PROFILE}

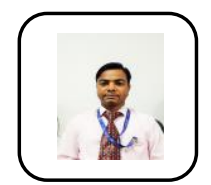

Dr. Om Prakash Sharma has joined as Assistant Professor to the School of IT, SRM University Sikkim. He obtained his PhD from SSSUTMS, Sehore, M.P. in 2019. He did his M.Tech in CSE from SMIT, Sikkim in 2012. He is also awarded with UGC-NET (Assistant Professor) in 2015. Before joining to this University, he has worked with VidyaVihar Institute of Technology, Purnea as a Sr. Lecturer in the Computer Science \& Engineering Department. His area of research interest includes Machine-Learning, Image-Processing, Biometrics based Security and IOT. He has published many papers in popular indexed journals.

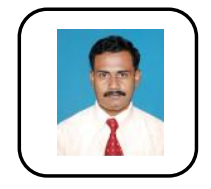

Mr.P.Sivaramkumar is working as an Assistant Professor in School of Information Technology, SRM University, Sikkim. He completed Bachelor of Engineering in Information Technology from Annamalai University. He completed Masters in Information Systems from Central Queensland University, Sydney, Australia. He is in the teaching field for more than a Decade. His area of interest is Network Security, Artificial Intelligence, Android and Web Development. 